\title{
Discussion to the papers by C. N. Pulvertaft, D. B. Morgan and G. R. Thompson
}

\author{
Chairman: Professor P. Fourman
}

Chairman. Thank you very much indeed, Dr Thompson for your stimulating, tantalizing exposition, on which I forbear to comment except to say that there are many reasons for visiting Bordier in Paris and to these can now be added the study of the calcification front.

Now, I would like to invite free discussion of these three papers, would somebody like to start the ball rolling?

WATKINSON. May I ask what you think of the suggestion that most of these post-gastrectomy patients with defective absorption of vitamin D were living in dingy basement flats?

THOMPSON. What you mean is, what is the role of sunlight in the whole problem? I think it is extremely difficult to assess. I don't think anyone has done any studies to determine what happens to people's vitamin D levels when they have more sun than others but I just think that it may be one of the possible factors concerned with the production of osteomalacia in some patients, and it does seem to be very much a factor which is important, certainly in London.

Chairman. In other words, many factors could be additive?

THOMPSON. I think so, yes.

HAMER. Have you been able to demonstrate any relations between the serum alkaline phosphatase and the amount of the stomach which is removed? Some people do a two-thirds gastrectomy, some people take more, have you been able to deduce any relations?

Pulvertaft. I think this is an extremely difficult question to answer; there are so many factors which come into it; there's age, there's how much stomach removed and in only one group of our series have we got any comparative measurements of the gastric remnant.

HAMER. You studied total gastrectomies too?

Pulvertaft. No, we had only two, whom we can compare with our Visick cases, but there are all sorts of things to consider before one can be certain that a relationship exists.

Thompson. I wonder if I could ask Dr Morgan a question, do you have any figures to support the fact that the phosphatase is higher in elderly subjects with Polyagastrectomy. Does it increase with age?

MoRgan. Well we have our own finding that the average alkaline phosphatase increased with age. This was important when we were comparing vagotomy with partial gastrectomy. It was established by Klaasen and in Sheffield by Hobson who pointed out as others have that alkaline phosphatase has a log-normal distribution. He suggested $20 \mathrm{KA}$ Units as the upper limit of normal in the elderly.

Thompson. Could I ask Dr Pulvertaft whether, when you were doing your 7-day dietary studies did you measure the fat intake in these patients and did it differ?

Pulvertaft. Yes we did. The difficulty here is to separate out low and normal weights, which are abnormal and which are normal, and it is even more difficult to relate this to the thickness of bone. The difference in intake of fat was quite small approximately 120 and $140 \mathrm{~g}$ daily.

Chairman. Is it possible to say whether the intake of fat was unnaturally small in the half dozen patients with osteomalacia?

Pulvertaft. Well, we haven't done those patients, because we have concentrated here on men, and as you will remember we've had very few cases of osteomalacia in men.

Dawson. I would like to ask Dr Morgan on this business of normal limits of alkaline phosphatase with age, he mentioned that dietary factors in old people may induce mild osteomalacia. What happens when you give vitamin $\mathrm{D}$ to a group of old people with a raised level? Does it come down or is it resistant?

Morgan. Well, I don't know. We do know that we took some of our patients with alkaline phosphatase of 12 units or more and gave then vitamin $\mathrm{D}$ and nothing happened, but I don't know what the situation is in the elderly.

Dawson. One often meets patients with a raised alkaline phosphatase, a bit of bone pain. We certainly had three or four patients with no other cause but old age for frank Looser's zones, that got better on vitamin D and one wonders then if this is not a fairly frequent phenomenon.

MoRgAN. I think this is a very important point. I am sure that osteomalacia in the elderly is much more common than we appreciate. I wouldn't suggest that because the alkaline phosphatase is only 16 that we should do nothing about it in an elderly patient.

Chairman. The exact prevalence of osteomalacia in the elderly has not been measured in the same way as this type of population study but the orthopaedic surgeons are seeing a lot. Mr Chalmers in Edinburgh has just published an account of his findings in the Journal of Bone and Joint Surgery and I think he described something like thirty cases, most of which we would accept as osteomalacia, a very striking disease of old age now.

DeLliPIANI. Just a small point for Dr Thompson. The environmental conditions in the post-gastrectomy gastrointestinal tract are very different from the controls and for this reason people have cast aspersions on the interpretation of the Schilling test after gastrectomy. I certainly had a very bizarre result in a post-gastrectomy patient using isotope tests for calcium absorption when you compare the results with controls, and in view of this I wonder if Dr Thompson has any comments to offer to 
do with the justification of comparing vitamin $\mathrm{D}$ absorption in these post-gastrectomy patients with controls?

THOMPSON. The results were very similar in as much as the nature of the radioactivity which was absorbed into the plasma was very similar after post-gastrectomy as it was in the controls; about $70 \%$ of radioactivity was vitamin D. I think that you are suggesting perhaps that bacteria might in some way destroy the isotope.

DellipianI. Well some mechanism such as that, or something to do with intestinal mixing. Some of the results I get with calcium absorption in post-gastrectomies again are very similar to controls, and in fact one suspects that these patients may have impaired absorption. This is what $\mathrm{Dr}$ Nordin anyway suggested about my own figures, and I wonder if something similar might apply in this situation.

THOMPSON. I think it would be difficult to make any interpretation of the abnormality in the various disturbances mentioned but as far as the validity of the test is concerned there was no difference in the nature of radioactivity in the plasma of the two groups, or in the proportion of the water-soluble tritium in the urine so I don't think there was an excess of exchange tritium in the gastrectomy patients as compared with the controls.

WATKINSON. May I ask Dr Morgan for the frequency of other causes of raised phosphatase in his series such as Paget's disease, bone injuries and those sort of things? Secondly, does Dr Thompson think in his absorption studies that the administration of vitamin $D$ with normal food makes a difference, because this seemed to make a big difference to iron absorption when it was given in fasting conditions and when it was mixed with ordinary food. Is the test really physiological?

ThOMPSON. I don't know, but I should imagine, judging by the results we got in lymph-fistula rats, that one fact which is very important to determine absorption is the amount of fat which the patient takes with a meal. We always gave the test in the standard manner, with a meal. We have never given it in any other way. I don't know what effect other food would have so I think it may be very important, one may find that if one uses this test on a fasting patient one may get a lower absorption.

DELLIPIANI. Following on from my previous question, I may have missed this but has Dr Thompson carried out the vitamin D absorption test on patients with osteomalacia not due to post-gastrectomy? What happens then?

THOMPSON. In the patients who have osteomalacia due to coeliac disease there is nearly always no absorption, which is easily demonstrated with this test. Likewise the same sort of result in patients with pancreatic steatorrhoea who get bone disease, and, in biliary cirrhosis with bone disease, a complete lack of absorption occurs. We found also one patient had renal osteodystrophy and this patient had normal absorption which I think one might have expected.

Chairman. May I ask a question following that, remember the data of Howard, Thomas and Morgan on the plasma and vitamin $D$ levels in various conditions including sprue, where they reported normal levels in sprue with osteomalacia. Do you have any explanation for that and does this accord with your data? These were assays.

ThOMpson. Yes, I've not done any studies on this but
I think it is a very interesting problem and I think, for vitamin $D$ to work you perhaps need more than just vitamin D in the plasma, I think certainly as far as the absorption of calcium is concerned, for example, the effect of vitamin D in promoting the synthesis in the carrier protein, then you presumably need a normal mucosa to produce the carrier protein in response to vitamin $D$. If the mucosa is still abnormal as it may be in sprue, if vitamin $D$ is given it is possible that it may not work until a gluten-free diet has restored the mucosa to normal.

Chairman. This certainly fits in with clinical experience doesn't it?

THOMPSON. Yes.

Neale. Could I ask Dr Morgan a little more about the nature of the bone disease he described. He described of course very clearly osteomalacia and described also the difficulties there are in osteoporosis, I wonder if he would tell us a little bit about what seems to be the nature of the bone condition in which one finds excess amounts of osteoid. I think he has described this somewhere about a year ago in a paper in the Lancet.

Morgan. We have described a condition of thin osteoid seams covering a proportion of bone-surface which was greater than what we thought normal. We found that an excess of osteoid was more common in patients after gastrectomy than it was in patients who had not had gastrectomy. There was some evidence that this slight excess of osteoid was more common in those patients who had a raised alkaline phosphatase. Our only suggestion is that the excess osteoid was due to increased turnover of bone, that it may not represent early osteomalacia or indeed may have nothing to do with vitamin D.

Chairman. But you did give vitamin D did you not to someone with such a condition, and had another biopsy, or am I mistaken?

Morgan. We did this, but only I think in three patients and as far as we could tell there was no difference in osteoid.

Chairman. And we didn't have the means of measuring the calcification front. We still haven't.

NeAlE. I wonder if I could come back and ask you why you were so surprised that subclinical osteomalacia is not a condition which goes on to frank osteomalacia? After all, after partial gastrectomy one frequently finds patients with apparent vitamin $B_{12}$ deficiency, serum vitamin $B_{12}$ between 80 and 120 , and if one follows these patients closely they will carry on with that kind of level of serum vitamin $B_{12}$ for very many years. With vitamin $\mathrm{D}$ one might expect exactly the same kind of thing to occur, particularly as patients every summer presumably get a small increase in their vitamin D levels from sunlight.

Morgan. This isn't analagous to subclinical osteomalacia which I defined as a stage which went on to gross osteomalacia. As such it didn't appear in numbers of patients we have seen. This is why I said that subclinical osteomalacia as we defined it was in the large either uncommon or didn't go on. If we start regarding the condition as a common one which may progress then we'd better start thinking about treating all these patients. 
NEALE. I think we are in agreement. We have seen these patients, as you have, and we have not seen one develop frank osteomalacia.

Chalrman. Another point that I had hoped $\mathrm{Dr}$ Morgan would make and I'll make it for him if he'll allow me to, is that we can't count very much on any form of sunlight in this country, whether in London or in Leeds because of the latitude. Dr Paterson, have you any comment to add?

Paterson. Of the twenty-two patients or so that Dr Morgan mentioned, certainly seven of them couldn't have had lack of sunlight as a factor in the formation of their osteomalacia as they lived mostly in the pleasant countryside around York.

Chairman. If York sunlight is any good, even in the countryside. Children in Britain all used to have rickets until they got vitamin D. I don't know if this sunlight is much help.

Dellipiani. Surely there was some work in Aberdeen, in sheep, showing that vitamin D level rises when there are adequate amounts of sunlight even though the animals were fed diets.

Chairman. I don't remember this work, I am ashamed to say, but was this sunlight or ultraviolet light?

Dellipiani. No this was sunlight.

Hancock. May I ask Dr Pulvertaft, the difference in bone thinning between the vagotomy gastro-enterostomy group and the post-gastrectomy group. This was based on a 5-year follow-up in the case of vagotomy and gastro-enterostomy as against a 10-year plus follow-up with gastrectomy. Surely it should be easy to get this type of figure on older people who have had their vagotomy and gastro-enterostomy before. The first question is has that been done? And secondly, if in fact there isn't this change in bone thinning with vagotomy and gastroenterostomy have you got any ideas why this should be so, is it in fact a mixing defect after gastrectomy? A lack of mixing food with the afferent duodenal loop leading perhaps to some failure of fat absorption leading to vitamin D deficiency?

Pulvertaft. Well you've got two questions. Our data on vagotomy and gastro-enterostomy is at this stage incomplete, we've only got follow-up periods up to 6 years, and a lot of data we have relates to 2 or 3 years after operation, so we don't know what will happen in the long run. We can't at this stage say that we won't get any metabolic bone disease later, we don't know. It is only in these early stages that we know there is no effect.

Now the nutritional side of gastrectomy, in effect, I simply don't know the answer to and I don't think anyone knows the answer either. It is a most peculiar thing. If you maintain a good state of nutrition you will get no bone change up to the age of 70 , if you don't the bones seem to go, but why this should be so I don't think we'd be prepared to discuss, whether this is again diet or absorption. We haven't got the answer. But certainly it is doubtful if diet is the complete answer and some patients seem to have adequate vitamin $D$ but still they've got thin bones.

Chairman. Dr Morgan, any questions? He won't be drawn. Peace has descended on the warring factions. Any other questions?

WATKINSON. Could you answer my previous question? How frequently could you explain the raised alkaline phosphatase in other ways?

MORGAN. In nearly all the patients with an alkaline phosphatase of 17 or more KA Units we could find the cause. In this range Paget's was twice as common as osteomalacia as a cause. If we took an alkaline phosphatase of between 12 and 17 then usually we'd fail to find a cause. We don't know what causes this minor increase in alkaline phosphatase but it did not respond to vitamin D.

Pulvertaft. May I add to that? It is the 11-17 unit group which is the fascinating group that we cannot explain, we suggested originally that this was intestinal alkaline phosphatase but further studies with Michael Langman didn't support this at all, but it does appear to be blood group related, there is more group $O$ in this particular group than there should be. Why we don't know.

ThOMPson. Finally, could I just postulate that at least some of these patients with a phosphatase between 12 and 17 have a degree of vitamin $\mathrm{D}$ deficiency and this is why their phosphatases are raised. These patients probably have an adequate proportion of calcification of the osteoid and it is, therefore, perhaps within the limits of these circumstances of calcium and bone. Whether these people will go on to osteomalacia I don't know.

MORGAN. I'd agree with this, that it is possible that some of these patients were as you say. But the point is that alkaline phosphatase of this level was just as common after vagotomy as it was after gastrectomy, and the patients with vagotomy had had the operation within the past 5 years. The frequency of this abnormality did not increase with time after the operation. Now if you were talking about vitamin D deficiency I would at least expect this to become more common with time after the operation. But I agree that within this group there might be a few patients as you describe.

Pulvertaft. May I come back on that? This is a fascinating group but how far should you go? You just find a raised alkaline phosphatase, should one treat them or not; or submit them to a lot of investigations. We have had one patient with an alkaline phosphatase of 12 initially which rose to abnormal levels after giving vitamin $D$, up to 20 , then it came down.

Thompson. But you had shown that some of these patients had thin bones and it may be that the thinning is a result of vitamin $\mathrm{D}$ deficiency.

Chairman. I think there is no disagreement with the form of words that Dr Thompson very carefully chose to say that some of these patients certainly have vitamin D deficiency, we just don't know how many. But we will emphasize this, and you will I am sure agree that the ones whom it is due to vitamin $\mathbf{D}$ deficiency should have a fall in the alkaline phosphatase some time after instituting treatment with vitamin $D$. It may rise at first but ultimately if it is due to vitamin $\mathbf{D}$ deficiency vitamin $\mathbf{D}$ should correct it; I am sure you will agree with this. The problem is how many. 